\title{
On the Horizontal Deviation of a Spinning Projectile Penetrating into Granular Systems
}

\author{
Waseem Ghazi Alshanti \\ Department of Mathematics and Statistics, University of Hail, Hail, Saudi Arabia \\ Correspondence should be addressed to Waseem Ghazi Alshanti; waseemalshanti@yahoo.com
}

Received 6 March 2017; Accepted 17 May 2017; Published 6 June 2017

Academic Editor: José-Ignacio Hidalgo

Copyright (C) 2017 Waseem Ghazi Alshanti. This is an open access article distributed under the Creative Commons Attribution License, which permits unrestricted use, distribution, and reproduction in any medium, provided the original work is properly cited.

\begin{abstract}
The absence of a general theory that describes the dynamical behavior of the particulate materials makes the numerical simulations the most current powerful tool that can grasp many mechanical problems relevant to the granular materials. In this paper, based on a two-dimensional soft particle discrete element method (DEM), a numerical approach is developed to investigate the consequence of the orthogonal impact into various granular beds of projectile rotating in both clockwise (CW) and counterclockwise (CCW) directions. Our results reveal that, depending on the rotation direction, there is a significant deviation of the $x$-coordinate of the final stopping point of a spinning projectile from that of its original impact point. For CW rotations, a deviation to the right occurs while a left deviation has been recorded for CCW rotation case.
\end{abstract}

\section{Introduction}

Any collection of many macroscopic discrete solids, whose typical size ranges from micrometers to centimeters, such that most of the particles are in contact with at least some of their neighboring particles, is called granular material. Sand, coal, sugar, corn, rice, and beads are classical examples for such materials. Granular materials exist almost everywhere in nature or any industry process. They are the base materials for most of the products, including food, agricultural, powder, pharmaceutical, mineral, and chemical products, and even the technology of missiles and penetrator bombs that burrow into ground to strike critical targets is affected by the properties of the grains within the ground. So far, there are still no reasonable theoretical justifications for many phenomena relevant to the particulate systems due to the rigorous mechanical behaviors of these systems. The dynamics of a granular bed impacted by a projectile penetrating at normal angle are still a challenging problem.

Even though the dynamics of penetration problem have long been of interest and remain an active research area attracting the attention of mathematicians and physicists, no consensus has emerged regarding the equation that describes the velocity of projectile during penetrating a granular bed [13]. Only a few studies have been conducted over the last three decades to better characterize the dynamics of penetration problem. Those studies, in general, focused on experimental investigations. In 1957, an equation was proposed which roughly expresses the negative acceleration of projectile penetrating randomly packed sand [4]. The equation has the form

$$
-\frac{d \vec{v}}{d t}=\alpha \vec{v}^{2}+\beta \vec{v}+\gamma,
$$

where $\alpha, \beta$, and $\gamma$ are positive constants and $\vec{v}$ denotes the projectile penetration velocity at time $t$. Also, it was suggested that the centrifuge modeling is an appropriate and powerful tool for investigating the penetration of projectile into granular soil [5]. For different soils, power relationships between projectile penetration depth and projectile mass-toarea ratio were presented. Moreover, the depth of penetration was proposed as a function of initial velocity and material properties of the granular bed [6]. A review of scaling laws for impact process and a brief for the current approaches that are used to study the processes of impacts was carried out $[7,8]$. Walsh et al. [9] performed experiments on impact craters 
formed by dropping a steel ball vertically into a container of small glass beads. By explicit variation of ball density, diameter, and drop height, the crater diameter was confirmed to scale as the fourth power of the energy of the ball at impact. More and more scaling laws that govern the penetration process of a projectile into granular beds were presented by a number of researchers [10-13]. Amato and Williams investigated the dependence of the crater diameter on the kinetic energy of falling ball into a sand filled container [14]. Bruyn and Walsh [15] reported that the penetration depth of a steel sphere dropped vertically into a container of loosely packed small beads increases linearly with the incident momentum of the projectile. The effect of several impact conditions on the rebound velocity of steel projectile was discussed in detail via three-dimensional (DEM) simulations [16]. It was shown that the impact velocity does not greatly affect the general scattering behavior of particulate aggregation but affects the rebound velocity of the steel projectile from the particulate aggregation. Several attempts have been made by using two- and three-dimensional (DEM) simulations to investigate deep penetration mechanism, impact crater, and force distribution of a projectile impacting into a granular medium [17-24]. The effect of closed lateral walls on penetration depth of a projectile has experimentally been studied $[25,26]$. A proposed model for the propagation of energy due to the impact of a projectile on a dense granular medium was established by Crassous et al. [27]. Further results can be found in $[28,29]$.

In the present work, based on this method, a mathematical model and numerical technique have been developed to study the dynamics of a rotating projectile impacting orthogonally into various granular beds. We investigate the effect of the rotation direction on the final position of the projectile after penetration.

\section{The Mathematical Model}

During simulation, granular particles bear two types of forces: contact forces and gravitational body force. Any contact force between two particles is decomposed into normal and tangential components. The normal contact force is modeled by a damped linear spring, while the tangential contact force is modeled by a linear spring in series with a frictional sliding element. The formula that determines the contact force of particle $i$ and particle $j$ is

$$
\vec{F}_{i j}(t)=\vec{F}_{i j, \widehat{n}}(t) \widehat{n}+\vec{F}_{i j, \widehat{s}}(t) \widehat{s}
$$

where $\widehat{n}$ and $\widehat{s}$ are unit vectors in the normal and shear directions of the contact plane and $\vec{F}_{i j, \widehat{n}}(t)$ and $\vec{F}_{i j, \hat{s}}(t)$ are, respectively, the magnitudes of the normal contact force and shear contact force; namely,

$$
\begin{aligned}
\vec{F}_{i j, \widehat{n}}(t)= & -k_{\widehat{n}} \vec{\delta}_{i j, \widehat{n}}(t), \\
\vec{F}_{i j, \widehat{s}}(t)= & -\operatorname{sign}\left[\vec{\delta}_{i j, \widehat{s}}(t)\right] \\
& \cdot \min \left\{k_{\widehat{s}}\left|\vec{\delta}_{i j, \widehat{s}}(t)\right|, \mu \vec{F}_{i j, \widehat{n}}^{e}(t)\right\},
\end{aligned}
$$

where $k_{\widehat{n}}$ and $k_{\widehat{s}}$ are, respectively, the particle-particle normal and tangential spring coefficients, $\vec{F}_{i j, \hat{n}}^{e}(t)$ and $\mu$ are, respectively, the elastic contribution of the contact force between the particles $i$ and $j$ in the normal direction ( $\widehat{n}$ direction) and the friction coefficient of the granular particles, $\vec{\delta}_{i j, \widehat{n}}(t)=$ $\left(R_{i}+R_{j}\right)-\left|\vec{r}_{i}(t)-\vec{r}_{j}(t)\right|$ and $\vec{\delta}_{i j, \hat{s}}(t)=\int_{t_{o}}^{t}\left(\dot{\vec{r}}_{i}(\eta)-\dot{\vec{r}}_{j}(\eta)\right) \cdot \widehat{s} d \eta$ are, respectively, the normal compression and the tangential displacement between the particles $i$ and $j$ over the time step $\Delta t=t-t_{o}$, and $R_{i}$ and $R_{j}$ are the radii of the particles $i$ and $j$. Under the contact forces and the gravitational body force, each particle has the following motion dynamic equations:

$$
\begin{aligned}
m_{i} \frac{d^{2} \vec{r}_{i}}{d t^{2}} & =\sum_{j=1}^{N_{p}} \vec{F}_{i j}+\vec{F}_{e i}, \\
I_{i} \frac{d^{2} \vec{\theta}_{i}}{d t^{2}} & =\sum_{j=1}^{N_{p}} \vec{M}_{i j}+\vec{M}_{e i},
\end{aligned}
$$

where $m_{i}, I_{i}, \vec{r}_{i}$, and $\vec{\theta}_{i}$ are, respectively, the mass, rotational moment of inertia, position, and rotational vectors of the centre of particle $i, \vec{F}_{i j}, \vec{M}_{i j}, \vec{F}_{e i}$, and $\vec{M}_{e i}$ are, respectively, contact force and moment acting on particle $i$ due to particle $j$ and external forces and moment acting on particle $i$, and $N_{p}$ is the number of particles within the granular bed. Hence, $\forall i=1,2,3, \ldots, N_{p}$, we have a system of first-order ordinary differential equations as follows:

$$
\begin{aligned}
& \dot{\vec{r}}_{i}(t)=\vec{v}_{i}(t), \\
& \dot{\vec{\theta}}_{i}(t)=\vec{\omega}_{i}(t), \\
& \dot{\vec{v}}_{i}(t)=\frac{\sum_{j=1}^{N_{p}} \vec{F}_{i j}+\vec{F}_{e i}}{m_{i}}, \\
& \dot{\vec{\omega}}_{i}(t)=\frac{\sum_{j=1}^{N_{p}} \vec{M}_{i j}+\vec{M}_{e i}}{I_{i}} .
\end{aligned}
$$

Therefore, by numerical integration of Newton's equation of motion, the updated velocities and positions of all particles can be determined.

\section{Numerical Simulation}

In 1979, Cundall and Strack [30] proposed the soft particle discrete method which is considered as one of the powerful tools for handling particulate systems. In the present work, for a typical simulation, a code was constructed by utilizing the $\mathrm{C}$ programming language. This code handles a two-dimensional discrete element computer simulation of circular particles subject to gravitational and contact forces. The code is able to model particles and walls with various properties, for example, contact properties, number of particles, size of particle, particle's density, and number of walls. A java movie code is also included so that the results of the simulations can be viewed. Consequently, a granular bed of $100 \times 50 \mathrm{~mm}$ dimensions is generated by the random 
TABLE 1: Parameter values used in simulation.

\begin{tabular}{lc}
\hline Parameter & Value (normalized) \\
\hline Number of particles within multisized bed, $N_{p}$ & 2200 \\
Number of particles within monosized bed, $N_{p}$ & 6400 \\
Particle's diameter within multisized bed, $d_{\text {multi }}(\mathrm{mm})$ & $0.9-2.0$ (uniform distribution) \\
Particle's diameter within monosized bed, $d_{\text {mono }}(\mathrm{mm})$ & 0.9 \\
Particle's density within multisized bed, $\rho_{\text {multi }}\left(\mathrm{kg} / \mathrm{m}^{3}\right)$ & 1 \\
Particle's density within monosized bed, $\rho_{\text {mono }}\left(\mathrm{kg} / \mathrm{m}^{3}\right)$ & 1 \\
Projectile's diameter, $d_{p}(\mathrm{~mm})$ & 6 \\
Projectile's density, $\rho_{p}\left(\mathrm{~kg} / \mathrm{m}^{3}\right)$ & 3000 \\
Projectile's impact velocity, $\vec{v}_{\text {imp }}(\mathrm{m} / \mathrm{sec})$ & 30 \\
Time step, $\Delta t(\mathrm{~s})$ & $5.37 \times 10^{-6}$ \\
\hline
\end{tabular}

TABLE 2: Mechanical properties of particles.

\begin{tabular}{lc}
\hline Property & Value (normalized) \\
\hline Particle-particle normal spring stiffness, $k_{n, \mathrm{pp}}(\mathrm{N} / \mathrm{m})$ & $1.266 \times 10^{5}$ \\
Particle-particle normal dashpot coefficient, $c_{n, \mathrm{pp}}(\mathrm{N} / \mathrm{m})$ & $2.524 \times 10^{2}$ \\
Particle-particle tangential spring stiffness, $k_{s, \mathrm{pp}}(\mathrm{N} / \mathrm{m})$ & $1.266 \times 10^{5}$ \\
Particle-particle friction coefficient, $\mu_{\mathrm{pp}}$ & 1.0 \\
Particle-wall normal spring stiffness, $k_{n, \mathrm{pw}}(\mathrm{N} / \mathrm{m})$ & $1.566 \times 10^{5}$ \\
Particle-wall normal dashpot coefficient, $c_{n, \mathrm{pw}}(\mathrm{N} / \mathrm{m})$ & $2.01 \times 10^{2}$ \\
Particle-wall tangential spring stiffness, $k_{s, \mathrm{pw}}(\mathrm{N} / \mathrm{m})$ & $3.006 \times 10^{4}$ \\
Particle-wall friction coefficient, $\mu_{\mathrm{pw}}$ & 1.0 \\
\hline
\end{tabular}

packing method where the particles are recognized as twodimensional discs. To study the influence of the particle size on the ultimate position of the rotating projectile after penetration, monosized particle and multisized particle beds with equal dimensions are constructed. Each granular bed is subjected to normal impact of projectile with diameter $d_{p}=6.0 \times 10^{-3} \mathrm{~m}$, density $\rho_{p}=3.0 \times 10^{3} \mathrm{~kg} / \mathrm{m}^{3}$, and fixed initial impact velocity $\vec{v}_{\text {imp }}=30 \mathrm{~m} / \mathrm{sec}$.

The simulation environmental conditions include physical properties of the granular particles under consideration, initial conditions, and boundary conditions, all being normalized. All parameters are normalized using the density of particle, gravitational acceleration, and particle diameter. A list of used normalized environmental and mechanical simulation parameters is given in Tables 1 and 2 .

\section{Results and Discussion}

In a normal penetration process, to analyze the consequences of the rotation's direction of the projectile on its final horizontal position with respect to the original impact point, a series of simulations are conducted with different considerations. The method is to vary both magnitude and direction of the projectile's angular impact velocity and keep all other simulation's parameters identical including the impact velocity $\left(\nu_{\text {imp }}=30 \mathrm{~m} / \mathrm{sec}\right)$. Figure 1 shows snapshots of the present numerical simulations in which the projectile orthogonally penetrates two similar $(100 \times 50 \mathrm{~mm})$ granular beds with different particle's size. The two granular beds are multisized and monosized particle beds. The range of particle's diameter within the multisized particle bed is $0.9 \mathrm{~mm} \leq d_{\text {multi }} \leq$ $2.0 \mathrm{~mm}$ and within the monosized particulate bed is equal to $d_{\text {mono }}=0.9 \mathrm{~mm}$.

For multisized particle bed, the projectile is given different CW and CCW angular velocities; namely, $\vec{\omega}=0$, $500,-500,1000$, and $-1000 \mathrm{rad} / \mathrm{sec}$. Then it is allowed to impact the middle of the free surface of the multisized granular bed with $\vec{v}_{\text {imp }}=30 \mathrm{~m} / \mathrm{sec}$. Figure 2 (a) shows the simulations results for each different value of the proposed angular velocities. The trajectory profile of the projectile exhibits two different regimes. In general, for CW angular velocities, namely, $\vec{\omega}=-500$ and $-1000 \mathrm{rad} / \mathrm{sec}$, the penetration trajectories of the projectile under the granular bed exhibit negative exponential-like traces. Conversely, positive exponential-like traces have been recorded for CCW angular velocities; namely, $\vec{\omega}=500$ and $1000 \mathrm{rad} / \mathrm{sec}$. Moreover, it is found that when the projectile comes to rest after achieving its maximum penetration depth, there is a considerable deviation for the horizontal $x$-coordinate of its ultimate stopping point from that of its original impact point. For CW rotations, namely, $\vec{\omega}=-500$ and $-1000 \mathrm{rad} / \mathrm{sec}$, a deviation to the right under the original impact point occurs while a left deviation happens for CCW rotation; namely, $\omega=500$ and $1000 \mathrm{rad} / \mathrm{sec}$. The case when the projectile has no angular velocity, that is, $\vec{\omega}=0 \mathrm{rad} / \mathrm{sec}$, the resultant projectile's trajectory is located in the middle of the two regimes' traces and the ultimate penetration point of the projectile is located, approximately, under its original impact point at the bed's surface. Therefore, it can be considered as a turning value angular velocity between the two regimes. 

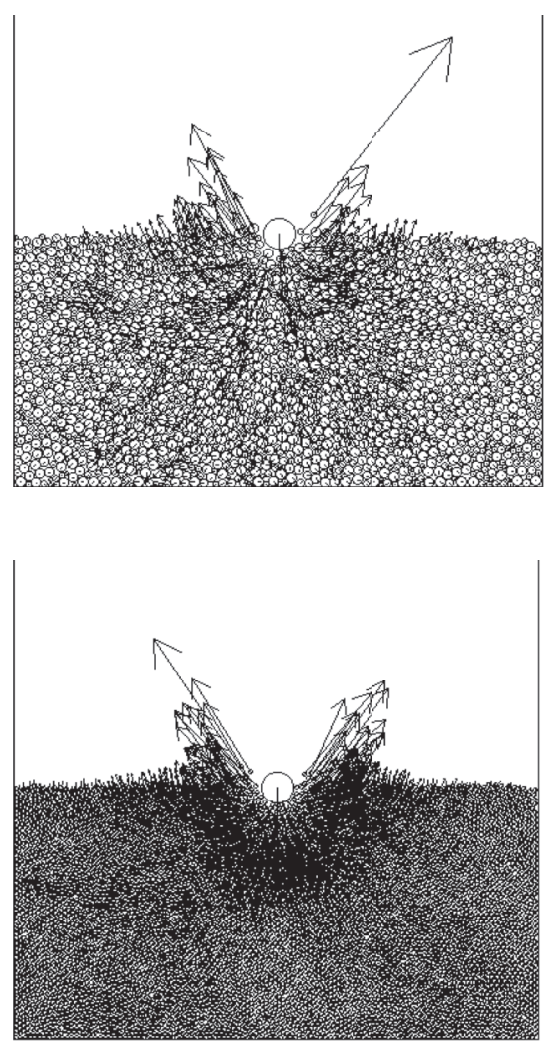

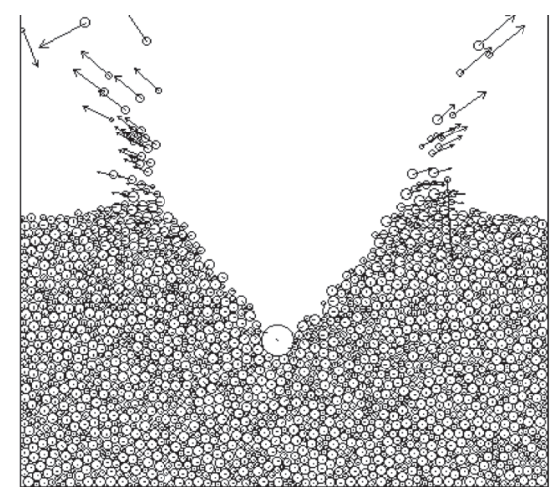

(a)

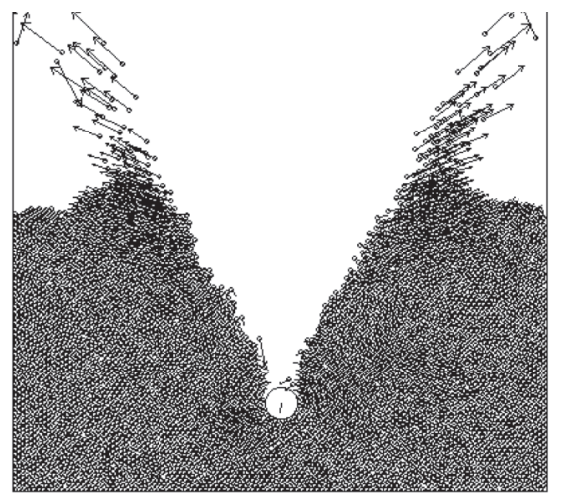

(b)
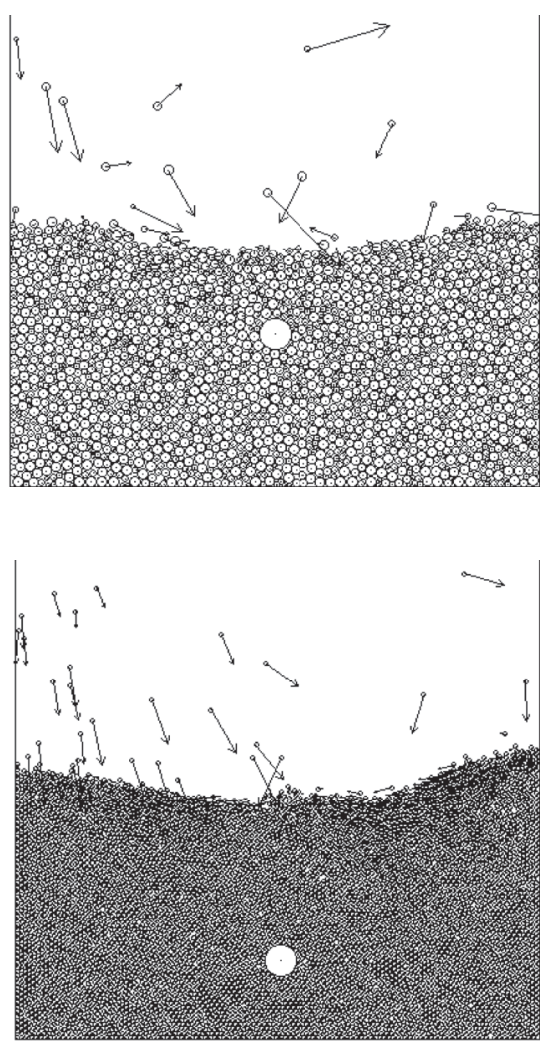

Figure 1: Simulation sequential snapshots of normal penetration process at, respectively, $t=0.2 \mathrm{sec}, 4.6 \mathrm{sec}$, and $16.4 \mathrm{sec}$. (a) Multisized particle bed. (b) Monosized particle bed.

A further similar series of simulations are carried out for the monosized particle bed with $d_{\text {mono }}=0.9 \mathrm{~mm}$. Again, all simulation's conditions are kept the same while the projectile is given different $\mathrm{CW}$ and CCW angular velocities; namely, $\vec{\omega}=0,500,-500,1000$, and $-1000 \mathrm{rad} / \mathrm{sec}$. The same phenomenon is observed as it was in the multisized particle bed as shown in Figure 2(b). For the CW angular velocities case, namely, $\vec{\omega}=-500$ and $-1000 \mathrm{rad} / \mathrm{sec}$, the traces of the projectile's penetration are negative exponentiallike traces and the ultimate stopping point of the projectile is located to the right of its original impact point, while positive exponential-like traces have been obtained for CCW angular velocities, namely, $\vec{\omega}=500$ and $1000 \mathrm{rad} / \mathrm{sec}$, with left deviation in the projectile's ultimate stopping point from its original impact point. For $\vec{\omega}=0 \mathrm{rad} / \mathrm{sec}$, when the projectile is given no angular velocity, no considerable shift is observed for the ultimate penetration point from the original impact point.

However, for multisized and monosized particle beds simulations, one can observe two changes in the achieved penetration depth of the projectile and the horizontal deviation magnitude in the projectile's ultimate stopping point from its original impact point. Consequently, under identical simulation conditions, the bigger the size of the bed's particles, the lower the projectile's normal penetration depth. On the other hand, the bigger the size of the bed's particles, the higher the horizontal deviation values of the projectile's ultimate penetration stopping point from that of its original impact point as shown in Figures 2(a) and 2(b). These results are in good agreement with previous experimental and computational results $[31,32]$.

Now, based on the above analysis of the proposed normal penetration processes, a function that describes the trajectory profile of the spinning projectile beneath the granular bed can be introduced as follows:

$$
y= \begin{cases}\alpha_{1} e^{x / \beta_{1}}+\eta_{1}, & \omega>0, \\ \alpha_{2} e^{-x / \beta_{2}}+\eta_{2}, & \omega<0,\end{cases}
$$

where $\alpha_{1}, \beta_{1}, \eta_{1}, \alpha_{2}, \beta_{2}$, and $\eta_{2}$ are constants depending on the magnitude of the projectile's angular velocity as well as the mechanical and environmental simulation's parameters. Under our simulation conditions the values of these six parameters are listed in Table 3.

To investigate the reason behind the shifting phenomenon of the projectile's ultimate penetration point from its original impact point for CW and CCW angular velocities, we consider both the normal and the tangential forces acting on the projectile during penetration process. Under the various values of the projectile's angular velocities, Figures 3(a) and 3(b) show the distributions of the normal forces acting on the projectile penetrating, respectively, within the 
TABLE 3: The values of the constants: $\alpha_{1}, \beta_{1}, \eta_{1}, \alpha_{2}, \beta_{2}$, and $\eta_{2}$.

\begin{tabular}{|c|c|c|c|c|c|c|}
\hline $\begin{array}{l}\text { Angular velocity } \\
\omega(\mathrm{rad} / \mathrm{s})\end{array}$ & $\alpha_{1}$ & $\beta_{1}$ & $\eta_{1}$ & $\alpha_{2}$ & $\beta_{2}$ & $\eta_{2}$ \\
\hline \multicolumn{7}{|c|}{ Multisized particle bed, $0.9 \mathrm{~mm} \leq d_{\text {multi }} \leq 2.0 \mathrm{~mm}$} \\
\hline 1000 & $1.0935 E-26$ & 0.79409 & 28.47253 & - & - & - \\
\hline 500 & $9.0172 E-41$ & 0.52424 & 30.5142 & - & - & - \\
\hline-500 & - & - & - & $3.3121 E 31$ & 0.72271 & 28.55692 \\
\hline-1000 & - & - & - & $2.7186 E 25$ & 0.90583 & 28.39503 \\
\hline \multicolumn{7}{|c|}{ Monosized particle bed, $d_{\text {mono }}=0.9 \mathrm{~mm}$} \\
\hline 1000 & $7.5136 E-67$ & 0.32018 & 14.93408 & - & - & - \\
\hline 500 & $1.9353 E-90$ & 0.23785 & 15.51606 & - & - & - \\
\hline-500 & - & - & - & $3.8043 E 110$ & 0.19916 & 15.44275 \\
\hline-1000 & - & - & - & $9.9635 E 35$ & 0.62992 & 15.27969 \\
\hline
\end{tabular}

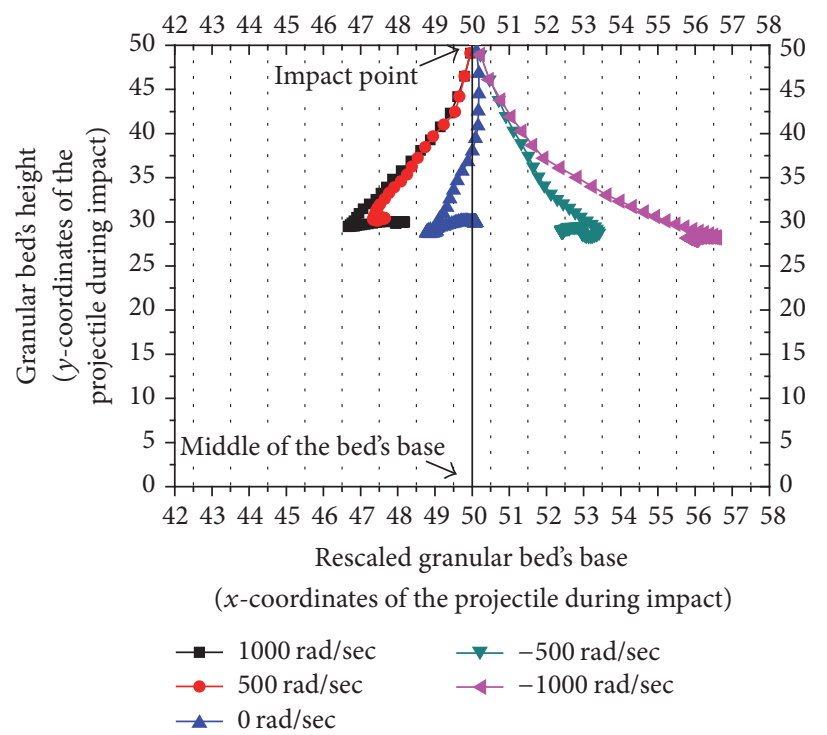

(a)

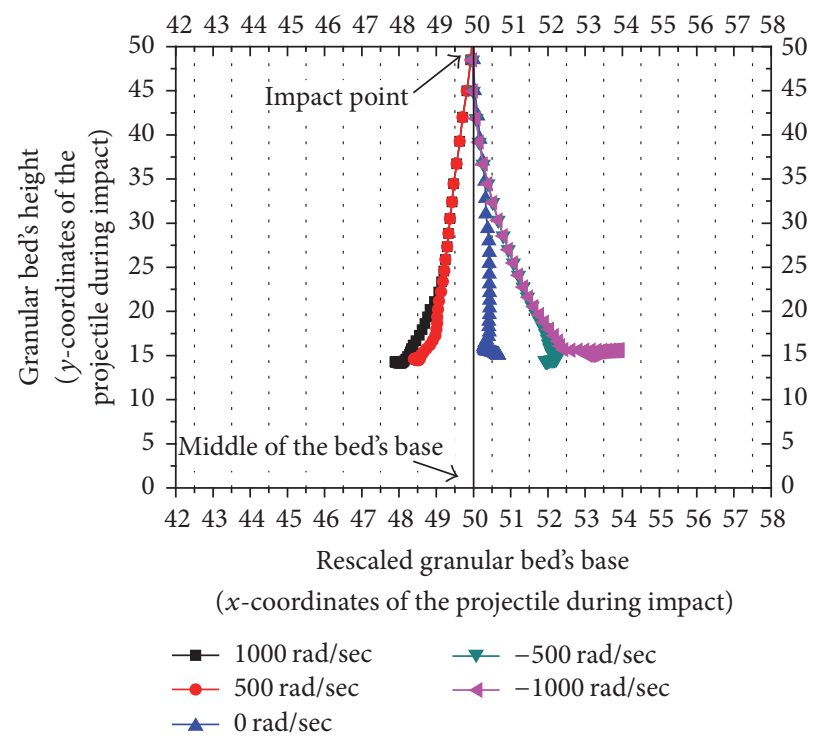

(b)

FIGURE 2: Deviation of the projectile's $x$-coordinate ultimate stopping point from that of its original impact point for various values of angular velocities. (a) Multisized particle bed. (b) Monosized particle bed.

multisized and monosized particle beds during the interval time $t=[0,10] \mathrm{sec}$.

Approximately, the profiles of the normal forces acting on the projectile at different values of angular velocities exhibit the same trace for both granular beds under consideration. Nevertheless, at the beginning of penetration process, relatively large oscillating has occurred in the normal force profile for the CCW angular velocities; namely, $\vec{\omega}=500$ and $1000 \mathrm{rad} / \mathrm{sec}$, while, after $t \approx 5 \mathrm{sec}$, all profiles exhibit the same trace. This indicates that the change in both the value and the direction of the angular velocity has no significant impact on the normal force distribution profiles.

The distributions of the tangential forces acting on the projectile penetrating the two granular beds over the same interval time are given in Figures 4(a) and 4(b).

A significant behavior of the tangential forces distribution has been observed in this case. For both granular beds, the tangential forces profiles exhibit relatively upwards oscillating for CW angular velocities; namely, $\vec{\omega}=-500$ and $-1000 \mathrm{rad} / \mathrm{sec}$, while, for CCW angular velocities, namely, $\vec{\omega}=500$ and $1000 \mathrm{rad} / \mathrm{sec}$, relatively downwards oscillating occurs in the tangential force profiles. This indicates that, for $\mathrm{CW}$ angular velocity penetrations, the right positive tangential forces, acting on the projectile, seem to dominate. Conversely, the left negative tangential forces are the dominant ones for the CCW angular velocities penetration processes. As a result, the dominant right positive tangential forces cause a relatively right shift in the horizontal position of the projectile during impact. Hence, for CW spinning penetrations, the projectile's ultimate final stopping point is located at the right to its original impact point. The converse happens when the projectile is given CCW angular velocities. Therefore, the various angular velocities of a projectile impacting a granular bed affect its ultimate horizontal penetration position. 


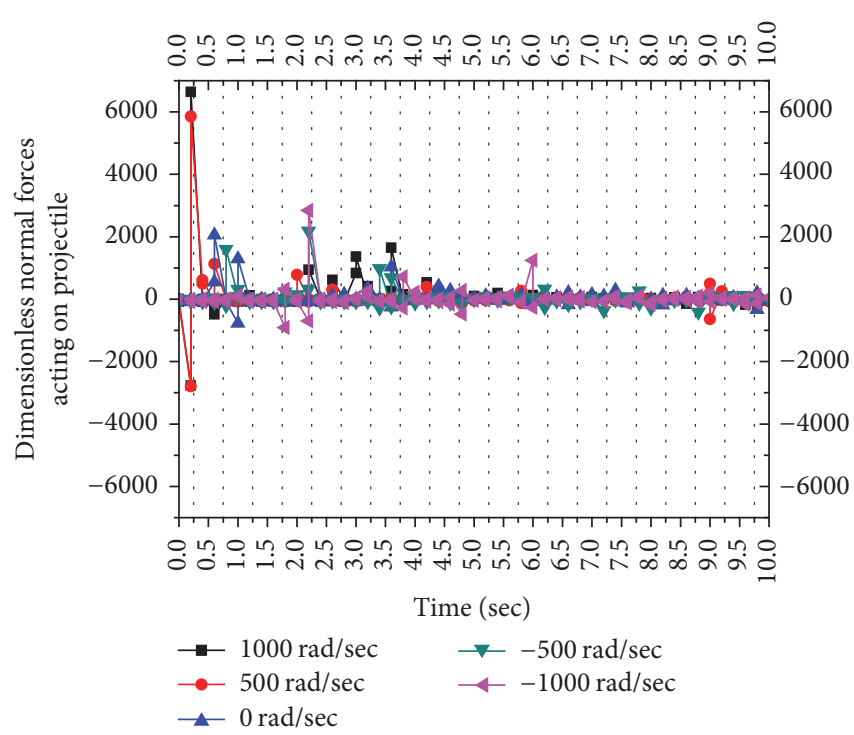

(a)

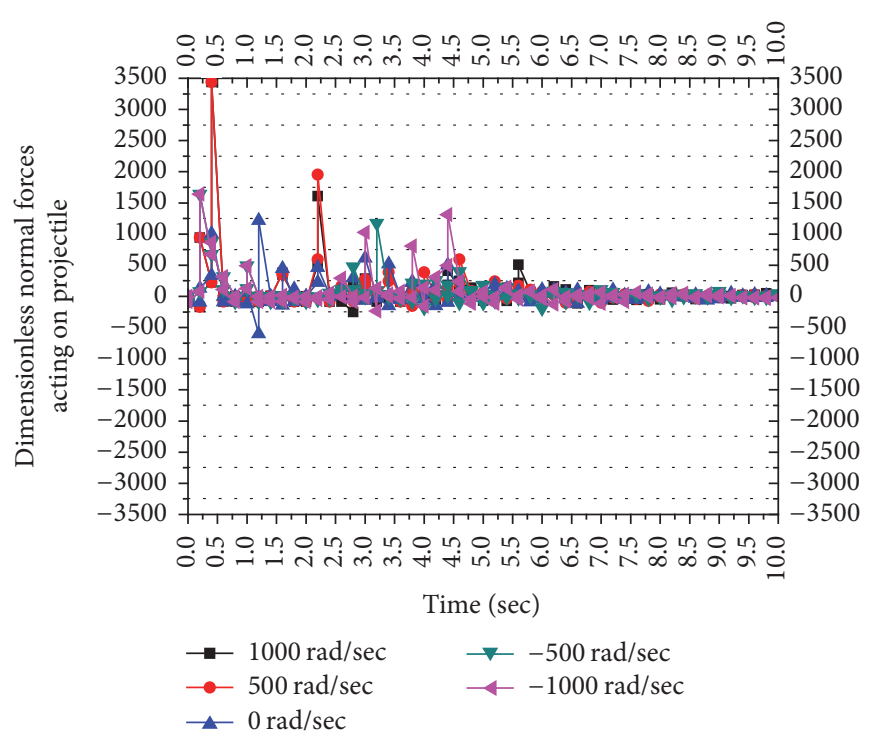

(b)

FIGURE 3: Normal forces acting on the projectile during penetration process. (a) Multisized particle bed. (b) Monosized particle bed.

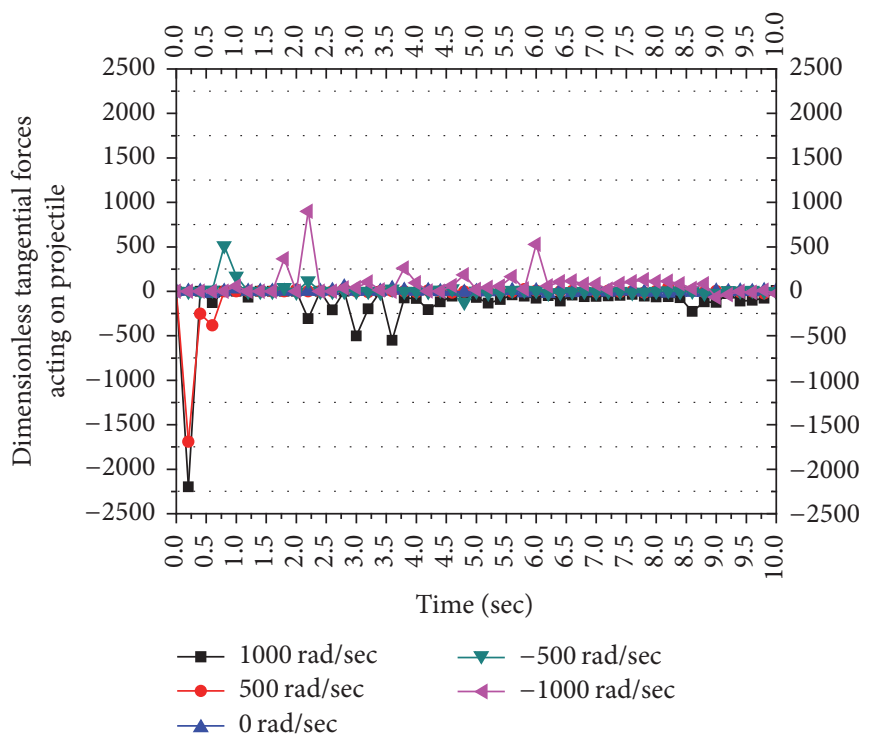

(a)

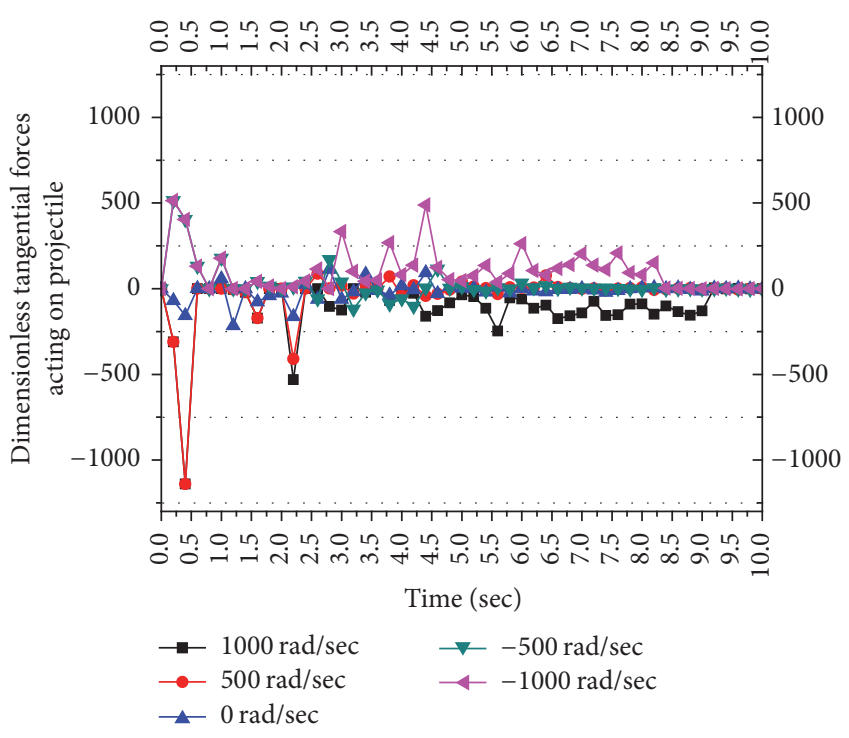

(b)

FIGURE 4: Tangential forces acting on the projectile during penetration process. (a) Multisized particle bed. (b) Monosized particle bed.

\section{Conclusion}

In the present work, a two-dimensional (DEM) mathematical model based on the soft particle method is established to investigate the trajectories of a normal spinning projectile equipped with various angular velocities to penetrate multisized and monosized granular systems. Our numerical results show that the model is able to simulate the normal penetration process for the various values of angular velocities. Moreover, it is found that the trajectory profile of the spinning projectile is affected by the magnitude as well as the direction of the projectile's angular velocity. Depending on the rotation direction of the projectile, there is a relatively small change on the ultimate horizontal position of the projectile after penetration. For CW angular velocities, a right shift on the ultimate penetration point from the original projectile's impact point is observed. On the other hand, the projectile's eventual penetration point is located to the left of its original impact point for the CCW angular velocities. A function that describes the trajectories of the spinning projectile at different angular velocities has been proposed.

\section{Conflicts of Interest}

The author declares no conflicts of interest. 


\section{References}

[1] M. Hou, Z. Peng, R. Liu et al., "Projectile impact and penetration in loose granular bed," Science and Technology of Advanced Materials, vol. 6, no. 7, pp. 855-859, 2005.

[2] M. Hou, Z. Peng, R. Liu, K. Lu, and C. K. Chan, "Dynamics of a projectile penetrating in granular systems," Physical Review E, vol. 72, article 062301, no. 6, Article ID 062301, 2005.

[3] D. Lohse, R. Rauhe, R. Bergmann, and D. Van Der Meer, "Granular physics: creating a dry variety of quicksand," Nature, vol. 9, no. 432, pp. 689-690, 2004.

[4] W. A. Allen, E. B. Mayfield, and H. L. Morrison, "Dynamics of a projectile penetrating sand," Journal of Applied Physics, vol. 28, no. 11, pp. 1331-1335, 1957.

[5] T. Taylor, R. J. Fragaszy, and C. L. Ho, "Projectile penetration in granular soils," Journal of Geotechnical Engineering, vol. 117, no. 4, pp. 658-672, 1991.

[6] Y. Boguslavskii, S. Drabkin, and A. Salman, "Analysis of vertical projectile penetration in granular soils," Journal of Physics D: Applied Physics, vol. 29, no. 3, pp. 905-916, 1996.

[7] K. A. Holsapple, "The scaling of impact processes in planetary sciences," Annual Review of Earth and Planetary Sciences, vol. 21, no. 1, pp. 333-373, 1993.

[8] H. Mizutani, "Scaling law on impact phenomena," Primitive Solar Nebula and Origin of Planets, pp. 297-317, 1993.

[9] A. Walsh, K. Holloway, P. Habdas, and J. de Bruyn, "Morphology and scaling of impact craters in granular media," Physical Review Letter, vol. 91, Article ID 104301, 2003.

[10] K. A. Newhall and D. J. Durian, "Projectile-shape dependence of impact craters in loose granular media," Physical Review E-Statistical, Nonlinear, and Soft Matter Physics, vol. 68, no. 6, Article ID 060301, pp. 603011-603013, 2003.

[11] M. A. Ambroso, C. R. Santore, A. R. Abate, and D. J. Durian, "Penetration depth for shallow impact cratering," Physical Review E-Statistical, Nonlinear, and Soft Matter Physics, vol. 71, no. 5, Article ID 051305, 2005.

[12] M. A. Ambroso, R. D. Kamien, and D. J. Durian, "Dynamics of shallow impact cratering," Physical Review E, vol. 72, article 04130, no. 4, 4 pages, 2005.

[13] D. I. Goldman and P. Umbanhowar, "Scaling and dynamics of sphere and disk impact into granular media," Physical Review E. Statistical, Nonlinear, and Soft Matter Physics, vol. 77, no. 2, Article ID 021308, 021308, 14 pages, 2008.

[14] J. C. Amato and R. E. Williams, "Crater formation in the laboratory: An introductory experiment in error analysis," American Journal of Physics, vol. 66, no. 2, pp. 141-143, 1998.

[15] J. R. Bruyn and A. M. Walsh, "Penetration of spheres into loose granular media," Canadian Journal of Physics, vol. 82, no. 6, pp. 439-446, 2004.

[16] M. Nishida, K. Tanaka, and Y. Matsumoto, "Discrete element method simulation of the restitutive characteristics of a steel spherical projectile from a particulate aggregation," JSME International Journal, Series A: Solid Mechanics and Material Engineering, vol. 47, no. 3, pp. 438-447, 2004.

[17] M. P. Ciamarra, A. H. Lara, A. T. Lee, D. I. Goldman, I. Vishik, and H. L. Swinney, "Dynamics of drag and force distributions for projectile impact in a granular medium," Physical Review Letters, vol. 92, no. 19, pp. 194301-1, 2004.

[18] L. Guerrero and L. Vallejo, "DEM Analysis of Crushing Around Driven Piles in Granular Materials," Geotechnique, vol. 55, no. 8, pp. 617-623, 2005.
[19] L. Tsimring and D. Volfson, "Modeling of impact cratering in granular media," in Proceedings of the Powders and Grains, vol. 2, pp. 1215-1223, Balkema, Rotterdam, 2005.

[20] K. Wada, H. Senshu, and T. Matsui, "Numerical simulation of impact cratering on granular material," Chemie Ingenieur Technik, vol. 42, no. 2, pp. 528-545, 2005.

[21] K. Wada, H. Senshu, and T. Matsui, "Numerical simulation of impact cratering on granular material," Icarus, vol. 180, no. 2, pp. 528-545, 2006.

[22] K. Wada, H. Senshu, S. Yamamoto, and T. Matsui, "A Numerical simulation of impacts into granular materials by distinctelement method, lunar and planetary science institute," vol. 34, 2003.

[23] F. Bourrier, F. Nicot, and F. Darve, "Physical processes within a 2D granular layer during an impact," Granular Matter, vol. 10, no. 6, pp. 415-437, 2008.

[24] M. J. Jiang, H.-S. Yu, and D. Harris, "Discrete element modelling of deep penetration in granular soils," International Journal for Numerical and Analytical Methods in Geomechanics, vol. 30, no. 4, pp. 335-361, 2006.

[25] S. B. Ogale, S. R. Shinde, P. A. Karve et al., "Impact-induced splash and spill in a quasi-confined granular medium," Physica A: Statistical Mechanics and its Applications, vol. 363, no. 2, pp. 187-197, 2006.

[26] A. Seguin, Y. Bertho, and P. Gondret, "Influence of confinement on granular penetration by impact," Physical Review E-Statistical, Nonlinear, and Soft Matter Physics, vol. 78, no. 1, Article ID 010301, 2008.

[27] J. Crassous, D. Beladjine, and A. Valance, "Impact of a projectile on a granular medium described by a collision model," Physical Review Letters, vol. 99, no. 24, Article ID 248001, 2007.

[28] J. R. Royer, E. I. Corwin, B. Conyers et al., "Birth and growth of a granular jet," Physical Review E-Statistical, Nonlinear, and Soft Matter Physics, vol. 78, no. 1, Article ID 011305, 2008.

[29] J. P. Borg and T. J. Vogler, "Mesoscale simulations of a dart penetrating sand," International Journal of Impact Engineering, vol. 35, no. 12, pp. 1435-1440, 2008.

[30] A. Cundall and O. Strack, "A discrete numerical model for granular assemblies," Geotechnique, vol. 29, no. 1, pp. 47-65, 1979.

[31] W. Alshanti and Y. Wu, "Numerical simulation of vertical penetration into granular beds," Australian Journal of Basic and Applied Sciences, vol. 5, no. 2, pp. 182-188, 2011.

[32] X. Ye, D. Wang, and X. Zheng, "Influence of particle rotation on the oblique penetration in granular media," Physical Review E-Statistical, Nonlinear, and Soft Matter Physics, vol. 86, no. 6, Article ID 061304, pp. 1-4, 2012. 

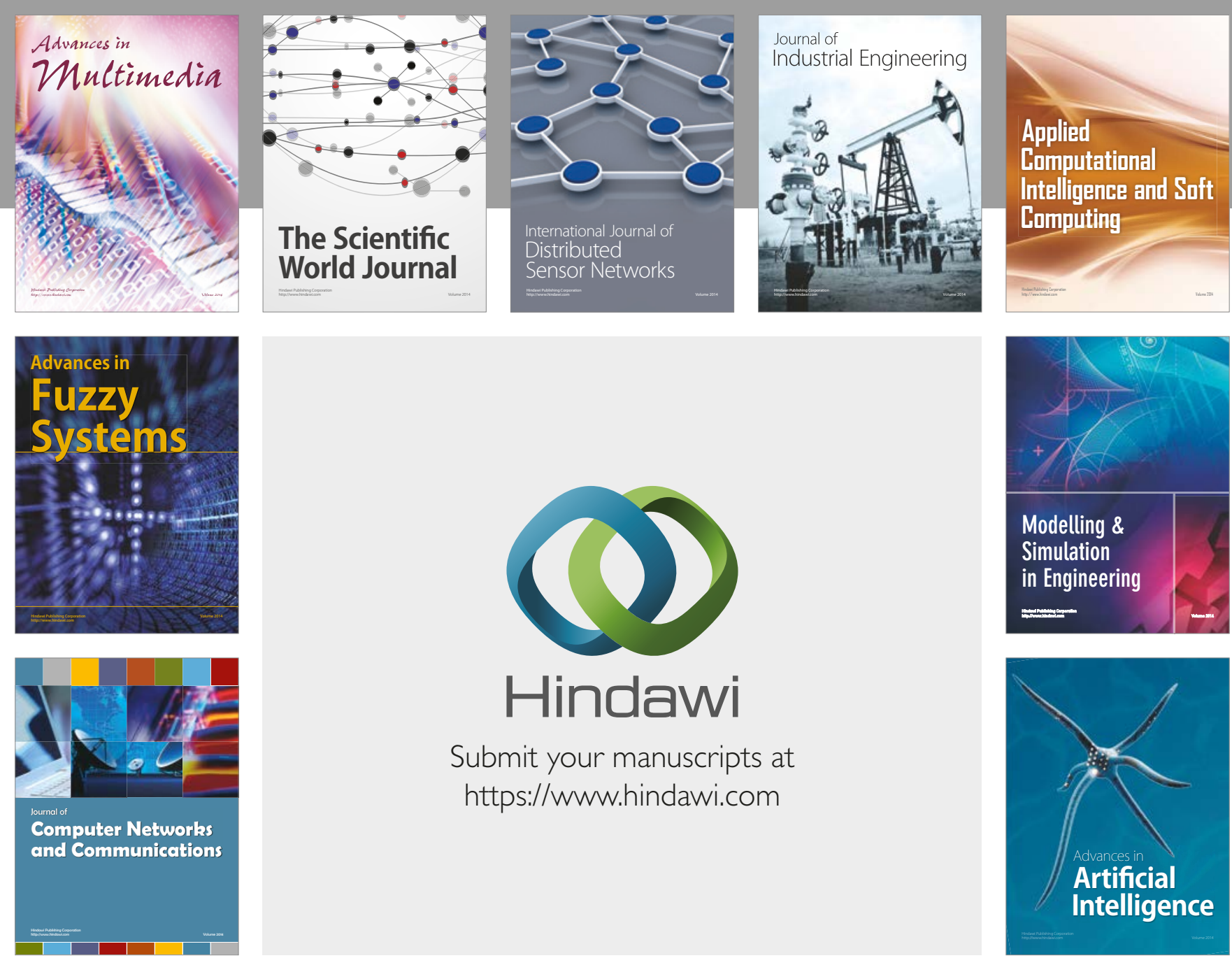

\section{Hindawi}

Submit your manuscripts at

https://www.hindawi.com
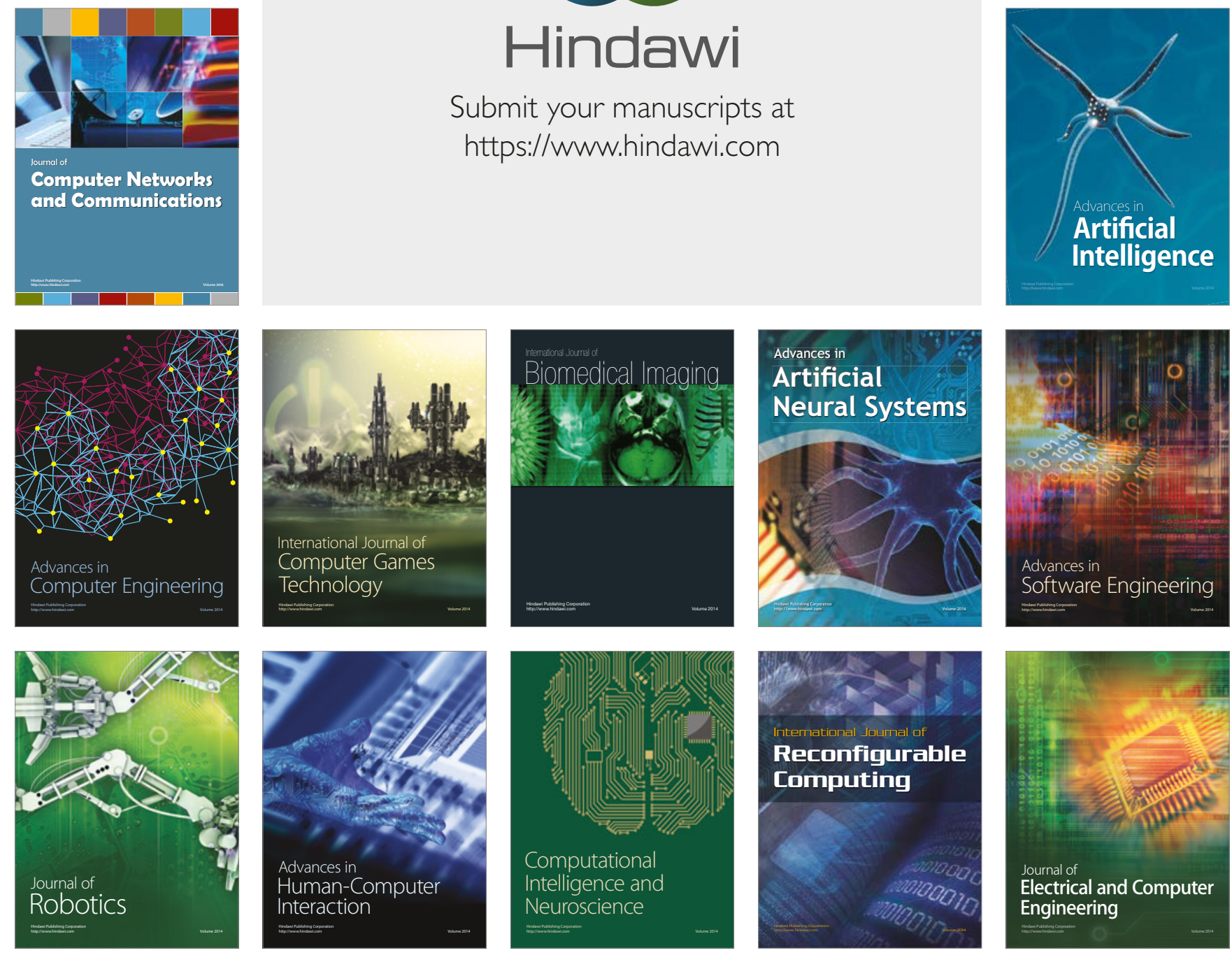